\title{
Dynamic Subchannel Assignment-Based Cross-Layer MAC and Network Protocol for Multihop Ad Hoc Networks
}

\author{
Khanh Nguyen Quang, ${ }^{1}$ Van Duc Nguyen, ${ }^{1}$ and Hyunseung Choo ${ }^{2}$ \\ ${ }^{1}$ School of Electronics and Telecommunications, Hanoi University of Science and Technology, Hanoi, Vietnam \\ ${ }^{2}$ Sungkyunkwan University, School of Information \& Communication Engineering, Republic of Korea \\ Correspondence should be addressed to Khanh Nguyen Quang; khanhnq1@vms.com.vn
}

Received 19 April 2013; Accepted 23 July 2013

Academic Editor: Youyun Xu

Copyright (c) 2013 Khanh Nguyen Quang et al. This is an open access article distributed under the Creative Commons Attribution License, which permits unrestricted use, distribution, and reproduction in any medium, provided the original work is properly cited.

\begin{abstract}
The paper presents a dynamic subchannel assignment algorithm based on orthogonal frequency division multiple access technology operating in the time division duplexing and a new cross-layer design based on a proposed routing protocol jointed with the MAC protocol. The proposed dynamic sub-channel assignment algorithm provides a new interference avoidance mechanism which solves several drawbacks of existing radio resource allocation techniques in wireless networks using OFDMA/TDD, such as the hidden node and exposed node problems, mobility, and cochannels interference in frequency (CCI). Besides, in wireless networks, when a route is established, the radio resource allocation problems may decrease the end to end performance proportionally with the length of each route. The contention at MAC layer may cause the routing protocol at network layer to respond by finding new routes and routing table updates. The proposed routing protocol is jointed with the MAC protocol based on dynamic sub-channel assignment to ensure that the quality of service in multihop ad hoc networks is significantly improved.
\end{abstract}

\section{Introduction}

The cochannel interference (CCI) is one of the major challenges in wireless networks for multihop communications. This interference is introduced when two different radio stations simultaneously use the same frequency. It is mainly caused by the spectrum allocated for the system being reused multiple times in TDMA network. CCI is one of the major limitations in cellular and personal communication services wireless telephone networks since it significantly decreases the carrier-to-interference ratio. In addition, it makes the diminished system capacity, more frequent handoffs, and dropped calls. IEEE 802.11 distributed coordination function operation is based on conventional carrier mechanism (CSMA/CA) in order to prevent channel collisions, CCI, and provide the communication between multiple pairs of independent mobile nodes without access points or base stations such as mobile ad hoc networks $[1,2]$.

Recently, orthogonal frequency division multiplexing (OFDM) has been intensively investigated for wireless data transmission in broadband cellular and ad hoc networks.
The multiple access technique for these networks is OFDMA [3]. The concept of this technique is to assign different users to different sub-channels in order to avoid interference.

Dynamic sub-channel assignment algorithm based on orthogonal frequency division multiple access (OFDMA) technology operating in time division duplexing has been studied in [4-7]. However, in most of the previous works, MAC layer is still transparent from physical layer.

In our previous work [7], we proposed a DSA algorithm based on orthogonal frequency division multiple access (OFDMA) technology operating in time division duplexing (TDD). This algorithm can maximize overall throughput of networks as well as solve some drawbacks existing in cellular networks such as hidden node and exposed node problems. However, in this work, a concept of multihop networks was not considered. The performance of the proposed DSA, therefore, was not presented in such networks. In this paper, we improve the DSA algorithm and apply it to the concept of multihop networks. In addition, we propose a simple routing protocol jointed with MAC model for multihop ad hoc networks based on OFDMA technology using 
the proposed DSA algorithm. The cost of a route in the proposed routing protocol refers to not only the distance but also the throughput of the route. By using the new concept of cost, the routing protocol aims to transmit data in a route with the balance of the distance and performance of the network in terms of throughput.

The paper is organized as follows. In Section 2, we briefly review sub-channels allocation methods. Section 3 describes the proposed DSA algorithm. In Section 4, the proposed routing protocol is presented. Simulation schemes, numerical results, are discussed in Section 5. Finally, conclusions are drawn in Section 6.

\section{Review of Subchannels Allocation Methods}

2.1. OFDM-FDMA Fix Allocation. The fixed allocation method of OFDM-FDMA for multiuser communications was proposed [8]. In such method, different users will be fixedly assigned to different sub-channels. Therefore, this method has not any anti-interference mechanism.

2.2. OFDM-FDMA Random Allocation. The OFDM-FDMA random allocation method is based on the idle and busy of sub-channels allowing users to account different subchannels [9]. However, it does not have any attention to the network interference. Once a sub-channel is selected, a user starts transmitting using the selected sub-channel. During a transmission process, if a sub-channel does not meet the required QoS, it will be released and assigned to a new user. Although the method is simple and it offers an adaptive mechanism, it does not provide CCI avoidance.

\section{Proposed DSA Algorithm}

Co-channel interference (CCI) is crosstalk from more than one different radio transmitter using the same frequency in wireless networks. Reducing CCI is very important since it makes the poor throughput performance. To avoid CCI and collisions, we propose a novel channel allocation algorithm called DSA which supports simultaneous transmissions in vehicle ad hoc network among nodes. In this section, the problem of CCI in OFDMA/TDD in wireless networks is discussed in detail. Then, we present the proposed dynamic sub-channel assignment (DSA) algorithm.

3.1. CCI in OFDMA/TDD System. To illustrate the problem of CCI, a simple scenario consisting of two base stations (BSs) and four mobile stations (MSs) is depicted in Figure 1. Let us assume an example of exchanging data among BSs and MSs as follows. The mobile station, $\mathrm{MS}_{1}^{\mathrm{Rx}}, \mathrm{MS}_{2}^{\mathrm{Rx}}$, and $\mathrm{MS}_{3}^{\mathrm{Rx}}$ receive data from base station $\mathrm{BS}_{1}^{\mathrm{Tx}}$, while at the same time the mobile station $\mathrm{BS}_{2}^{\mathrm{Tx}}$ transmits its data to the base station $\mathrm{MS}_{4}^{\mathrm{Rx}}$. In such scenario, $\mathrm{BS}_{2}^{\mathrm{Tx}}$ causes $\mathrm{CCI}$ to receive the data of $\mathrm{MS}_{1}^{\mathrm{Rx}}$, $\mathrm{MS}_{2}^{\mathrm{Rx}}$, and $\mathrm{MS}_{3}^{\mathrm{Rx}}$; the base station $\mathrm{BS}_{1}^{\mathrm{Tx}}$ causes CCI to receive the data of $\mathrm{MS}_{4}^{\mathrm{Rx}}$. Note that CCI only exists in TDD mode.

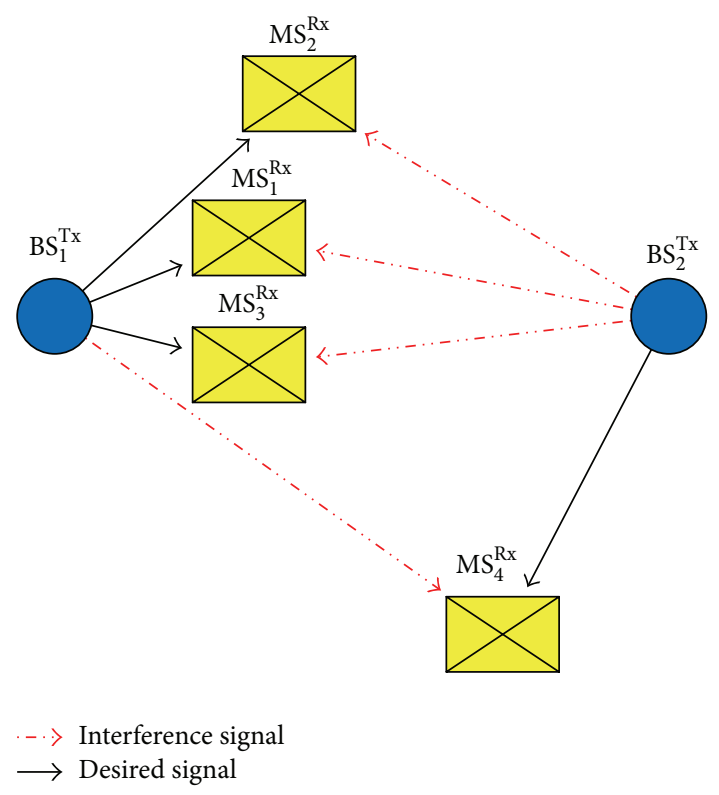

FIGURE 1: Cochannel interference in OFDMA/TDD system.

If a node can select appropriate sub-channels in available sub-channel set before the data transmission, CCI is minimized and thus increases the throughput of the network.

3.2. Subchannel Selection Based on Busy Signals. In the previous example, CCI was introduced, and it badly affects the receiving data of $\mathrm{MS}_{1}^{\mathrm{Rx}}, \mathrm{MS}_{2}^{\mathrm{Rx}}$, and $\mathrm{MS}_{3}^{\mathrm{Rx}}$. To avoid this interference, $\mathrm{MS}_{1}^{\mathrm{Rx}}, \mathrm{MS}_{2}^{\mathrm{Rx}}$, and $\mathrm{MS}_{3}^{\mathrm{Rx}}$ have to broadcast a busy signal when they receive data. Before data transmission, $\mathrm{BS}_{2}^{\mathrm{Tx}}$ will first hear busy signals in all channels and then compare the received signal power with a predetermined threshold. If the power of busy signal in a channel is lower than the threshold, $\mathrm{BS}_{2}^{\mathrm{Tx}}$ can select the channel for data transmission.

3.3. Dynamic Subchannel Assignment Algorithm. In this section, we propose an algorithm for downlink and uplink transmissions based on incorporation of the sub-channel selection based on busy signals mechanism in OFDMA/TDD network. The proposed algorithm is illustrated in Figure 3. The busy signal sent an in-band signaling before data transmission. Data sub-channels are mutually orthogonal and used for transmitting OFDM symbols.

Figure 2 shows data transmitting and receiving in view point from BS and MS, respectively [4]. The structure of the uplink frame is similar to that of the downlink frame. Each subframe includes a busy signal channel (an OFDM symbol) used for signaling busy signal. MAC frame length is chosen corresponding to the time correlation of the channel. Initially, the sub-channel is selected by only the transmitter. On the other hand, the adaptive period is adjusted by both transmitter and receiver. We define two kinds of signal:

(i) busy signal: transmitted only in a channel by MS receiver,

(ii) data signal: transmitted only by BS transmitter. 

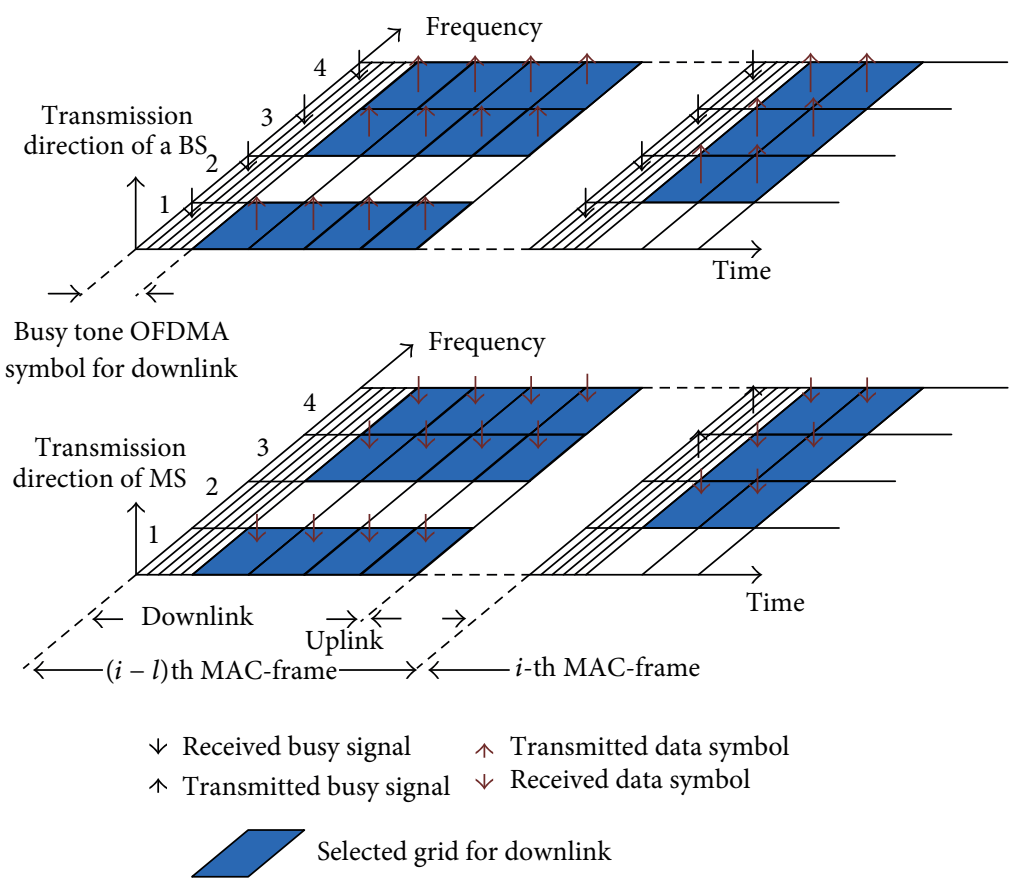

FIgURE 2: Structure of the MAC frame for DSA algorithm.

In Figure 3, the proposed algorithm includes the two following steps.

(1) Link Initialization. Link initialization occurs when $m$ th BS needs to transmit data to $k$ th MS in the network. Firstly, $m$ th BS listens to all busy signals and compares each of these busy signals with a given threshold to obtain a set of available subchannel for data transmission called set A. A sub-channels is available if the power of the received busy signal is below the threshold. The $m$ th BS will start transmiting its data to receiver using a set of available channel at the $(i-1)$ th MAC frame. We propose a mathematical model (1), where $a_{l, i-1}^{k, m}$ denotes the sub-channel assignment for $l$ th sub-channel at the $(i-1)$ th MAC frame of the link between $m$ th BS and $k$ th MS. If the sub-channel is assigned to the link between $m$ th BS and $k$ th MS, then $a_{l, i-1}^{k, m}=1$; otherwise $a_{l, i-1}^{k, m}=0$. The outcome of this channel assignment is obtained by comparing the busy signal with the threshold as follows:

$$
a_{l, i-1}^{k, m}= \begin{cases}1 & \text { if }\left|\widehat{B}_{l, i-1}^{m}\right| \leq I_{\mathrm{th}} \\ 0 & \text { otherwise. }\end{cases}
$$

$I_{\text {th }}$ is a threshold being a measure for the interference that this transmission would effect to other coexisting transmissions. Set A is all sub-channels having channel assignment by (1).

(2) Dynamic Subchannel Assignment. At the $(i-1)$ th MAC frame, $k$ th MS estimates the SINR on each sub-channel of set A. Based on the given QoS requirement for the transmission, $k$ th MS will decide to maintain or release the respective subchannel of set A. $k$ th MS will only broadcast the busy signal on the remained sub-channels. If the sub-channel is specified, then $b_{l, i-1}^{k, m}$ is assigned 1 otherwise $b_{l, i-1}^{k, m}=0$.

We propose a mathematical model (2), where $b_{l, i-1}^{k, m}$ denotes the reservation of $l$ th sub-channel for the $i$ th MAC frame. $\breve{\gamma}_{l, i-1}^{k}, \gamma_{\text {req }}$ are SINR estimated by $k$ th MS and required QoS,

$$
b_{l, i-1}^{k, m}=\left\{\begin{array}{l}
1 \\
0
\end{array} \text { if }\left(\left|\widehat{B}_{l, i-1}^{m}\right| \leq I_{\mathrm{th}}\right),\left(\breve{\gamma}_{l, i-1}^{k} \geq \gamma_{\mathrm{req}}\right) .\right.
$$

Since the ith MAC frame, the condition for the subchannel assignment on the desired or new link between $m$ th BS $\mathrm{v} k$ th MS for subsequent MAC frames is given as follows:

$$
a_{l, i}^{k, m}= \begin{cases}1 & \text { if }\left(\bar{a}_{l, i-1}^{k, m}\left|\widehat{B}_{l, i}^{m}\right| \leq I_{\text {th }}\right) \text { or } b_{l, i-1}^{k, m}=1 \\ 0 & \text { otherwise, }\end{cases}
$$

where $\widehat{B}_{l, i}^{m}$ is the busy signal received at the $m$ th BS on the $l$ th sub-channel at the $i$ th MAC frame and

$$
\bar{a}_{l, i-1}^{k, m}= \begin{cases}1 & \text { if } a_{l, i-1}^{k, m}=0, \\ 0 & \text { otherwise. }\end{cases}
$$

Since the $i$ th MAC frame, set A includes all maintained sub-channels (set B of $(i-1)$ th MAC frame) and new respective sub-channels if the power of their received busy signal is below the threshold.

\section{Proposed Routing Protocol Description}

In multihop ad hoc networks, a node transmits its data to a destination node via an optimal route. In order to find 


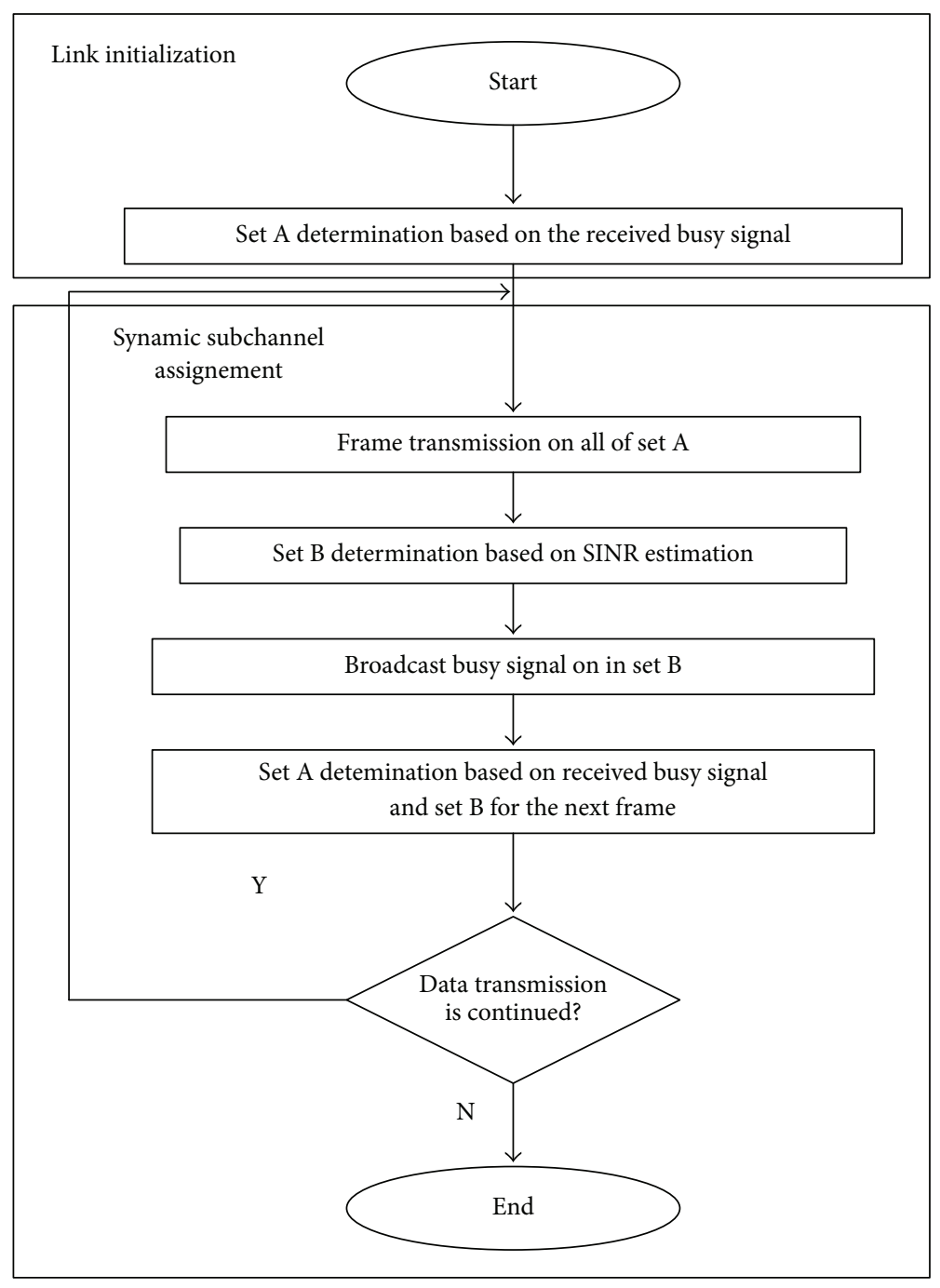

FIGURE 3: Flowchart of the proposed DSA algorithm.

the optimal route, the routing is implemented. In this section, we propose a new routing protocol which can be easily jointed to the MAC protocol based on the DSA algorithm. The corporation between the routing and the MAC protocol not only ensures the mobility and multihop but also gains the performance in terms of throughput in the multihop ad hoc networks.

The routing protocols working in wireless networks depend on the radius of coverage of nodes. The connection among all nodes changes fastly over time. The principal cost of the proposed routing protocol is the combination of the distance among nodes and the throughput of a route. The finding of the shortest route in the proposed protocol is based on the shortest route finding algorithms and the radius of coverage of nodes in the network. To ensure the mobility and the performance in terms of throughput of the network, the MAC provides the number of selected channels of a route to the routing protocol. This value is compared with a proposed threshold called Channel_threshold in order to update the optimal route for the transmission.
Before describing the flowchart of this protocol, we define the following.

(i) $R_{i}$ is the radius of coverage of $j$ th node in the network.

(ii) The network is supposed to be a graph with nodes and edges of the network.

(iii) The connection among nodes is described in a connection matrix called connection matrix.

We propose a mathematical model which is based on model (5) for the connection matrix:

$$
M_{i j}= \begin{cases}\operatorname{distance}(i, j) & \text { if distance }(i, j) \leq \min \left(R_{i}, R_{j}\right) \\ 0 & \text { otherwise }\end{cases}
$$

where $M_{i j}$ and distance $(i, j)$ are the connection state and the distance between node $i$ and node $j$, respectively. 


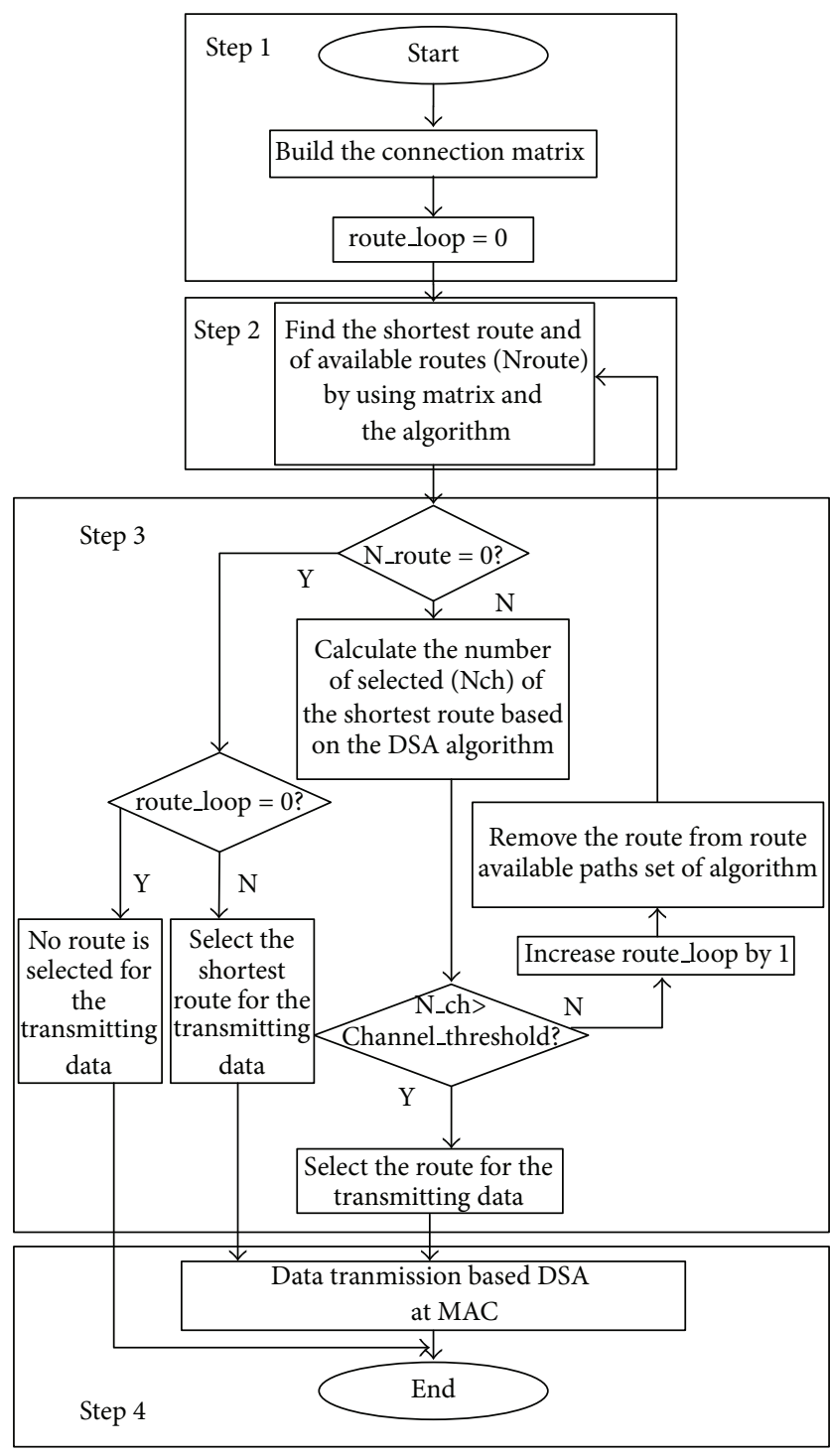

FIGURE 4: Flowchart of the proposed routing protocol.

In Figure 4, the proposed routing protocol finds the route using the following three steps.

Step 1. The input of this step is the radius of coverage of all nodes and the distances between all node pairs in the network. The connection matrix is built based on the proposed mathematical model (5). The value of init_loop is initially set 0 .

Step 2. In this step, the shortest route and the number of available routes (iNP) are obtained by using the Dijkstra algorithm and shortest route algorithm; see [10]. The inputs of the step are the positions of the destination and source node and the connection matrix.

Step 3. This step aims to find the optimal route for the transmitting data. Firstly, iNP is checked. If iNP is nonzero, there is at least one available route between the source node and the destination node. The number of selected sub-channels (iSC)
TABLE 1: OFDM system parameters.

\begin{tabular}{lc}
\hline Parameters & Values \\
\hline Bandwidth $(B)$ & $20 \mathrm{MHz}$ \\
Sampling interval $\left(t_{a}=1 / B\right)$ & $50 \mathrm{~ns}$ \\
FFT length $\left(N_{\mathrm{FFT}}\right)$ & 256 \\
OFDM symbol duration $\left(T_{S}\right)$ & $12.8 \mu \mathrm{s}$ \\
Guard interval $\left(T_{G}\right)$ & $2 \mu \mathrm{s}$ \\
Frequency $\left(f_{c}\right)$ & $1.9 \mathrm{GHz}$ \\
Modulation scheme & $16-\mathrm{QAM}$ \\
SINR $_{\text {min }}\left(\gamma_{\text {req }}\right)$ & $16 \mathrm{~dB}$ \\
Channel_threshold & 42 \\
\hline
\end{tabular}

of the shortest route is derived by using the proposed DSA algorithm. If iSC is larger than the given Channel_threshold, it means that the route ensures the requirement of throughput of the system. Consequently, the shortest route is selected as the optimal route for the transmission data. Otherwise, the route does not satisfy the requirement of the system. We, therefore, have to find another route. The init_loop is set to 1 which points that there is at least one available route not satisfying the requirement of throughput of the network. The step is directed to the beginning of Step 2, and the shortest route is excluded in the Dijkstra algorithm. At the end of Step 1, if iNP is zero, these two cases can happen: the first case is that there is no available route between the source node and the destination node (init_loop is 0 ); hence, the transmission is stopped and it has to delay for some time to transmit the data. In the other case, there is at least one route available. However, all of the nodes do not satisfy the requirement of throughput of the network. In this case the shortest node is selected for the transmission data.

Step 4. In this step, the data transmission based on the DSA algorithm at MAC layer will occur.

\section{Simulation Model}

In the simulation scheme, OFDM parameters are selected in Table 1. 16-QAM modulation is selected for all sub-channels. We will present the performance in terms of throughput of the networks of our proposed method in three scenarios. In the first scenario, the performance of the proposed DSA is demonstrated in single-hop ad hoc networks without the proposed routing protocol. Then, the proposed DSA algorithm and the proposed routing protocol are applied in the scenario of multihop ad hoc networks. However, in this case, the routing is implemented without selecting the optimal route in terms of throughput of the route. Finally, the proposed routing protocol that cooperated with the proposed MAC protocol based on DSA algorithm is fully carried out in multihop ad hoc networks.

\subsection{Throughput of Single Hop Ad Hoc Networks}

5.1.1. Analysis Model. We consider a model including two transmitter-receiver pairs depicted in Figure 5, where Tx and $\mathrm{Rx}$ are the transmission mode and the receive mode, respectively. TDD mode is used. In this model, the distance 


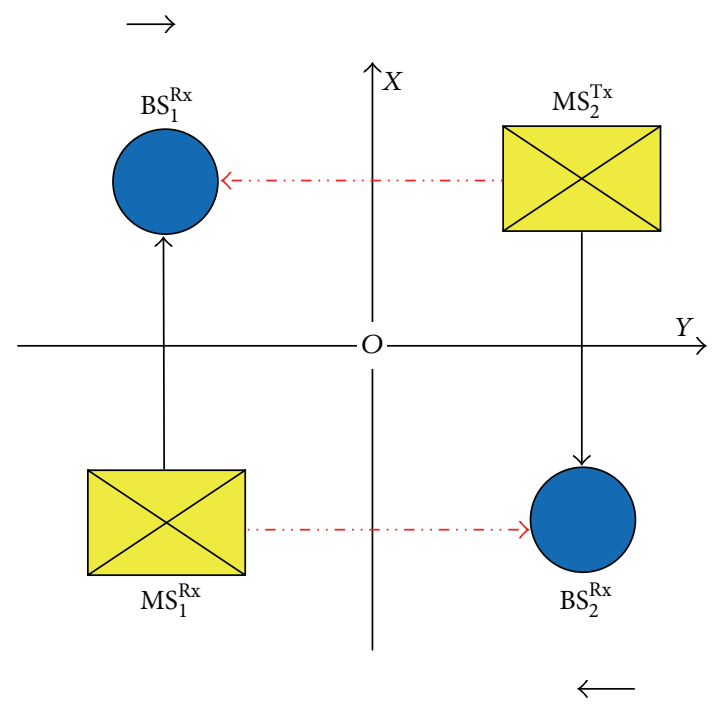

FIGURE 5: A simple circumstance for single-hop ad hoc network.

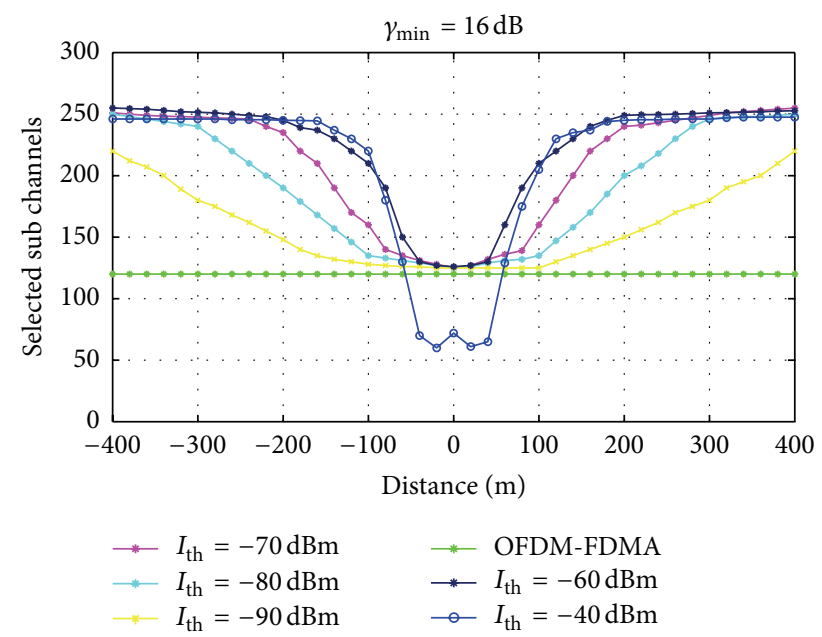

FIgURE 6: The number of selected sub-channels with different thresholds and conventional OFDM-FDMA.

between two nodes in each pair is constant, and these pairs move in the opposite direction.

5.1.2. Numerical Results. In this evaluation, the throughput in terms of the number of selected sub-channels is considered for two methods: OFDM-FDMA fixed allocation and proposed DAS algorithm. It can be seen from Figure 6 that the throughput of network using the proposed DSA depends on the value of different threshold $I_{\text {th }}$ as well as the distance between pairs in the network. When the distance between pairs is high, the proposed method demonstrates a high performance. Since in OFDM-FDMA fixed allocation method sub-channels are fixedly allocated to users, its throughput is constant and much less than the one of the proposed DSA method. On the other hand, the proposed method offers CCI avoidance mechanism based on busy signal. The selection of sub-channel consider to given threshold, thus the throughput

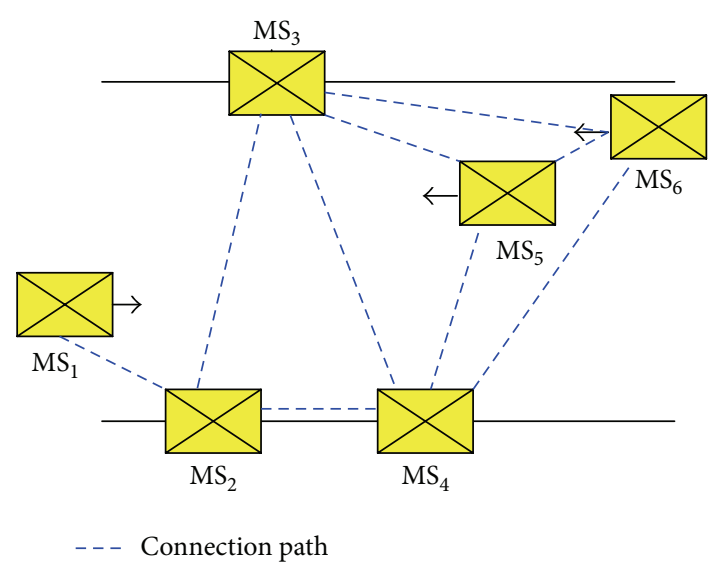

FIGURE 7: Initial state of networks used in simulation in the case of multihop ad hoc networks.

depends on the selection of threshold; From the results in Figure 6, the suitable threshold for the proposed method is $-60 \mathrm{dBm}$.

\subsection{Throughput of Multihop Ad Hoc Networks Based on Only the Proposed DSA Algorithm and MAC Structure}

5.2.1. Analysis Model. In this scenario, the performance of the proposed DSA algorithm and the MAC structure is evaluated in a multihop ad hoc network considering the effect of the length of selected routes and $I_{\mathrm{th}}$. Therefore, the routing protocol used in this model is not the proposed routing protocol presented in Section 4. It is just the principle routing which considers only the distance of a route as the cost of this route. The shortest route being selected as the optimal route for transmitting data is obtained by using the Dijkstra algorithm. The input of the Dijkstra algorithm is the connection matrix of the network. Before each transmission section, the connection matrix is built as proposed in Section 4. Then, the shortest route output from the Dijkstra algorithm is selected. The data will be transmitted through the selected route. We consider a vehicles network VANET that includes 6 mobile stations (MSs) indexed from 1 to 6 with the initial state depicted in Figure 7. $\mathrm{MS}_{1}, \mathrm{MS}_{5}$, and $\mathrm{MS}_{6}$ are running step by step while the other mobile stations are fixed. The running step length is set to 5 meters. $\mathrm{MS}_{5}$ and $\mathrm{MS}_{6}$ run in the same direction being opposite to the running direction of $\mathrm{MS}_{1}$. While the mobile stations are running, $\mathrm{MS}_{1}$ is transmitting its data to $\mathrm{MS}_{5}$. After each step, to throughput of whole network is obtained. Figure 8 gives an example of the connection state of the network. It can be seen from Figure 8 that the shortest route $\left(\mathrm{MS}_{1} \rightarrow \mathrm{MS}_{2} \rightarrow \mathrm{MS}_{4} \rightarrow \mathrm{MS}_{5}\right)$ is selected for the transmitting data.

5.2.2. Numerical Results. Figure 9 plots the length of route from $\mathrm{MS}_{1}$ to $\mathrm{MS}_{5}$ with respect to running steps. Since the routing protocol is not based on $I_{\text {th }}$, the length of routes for the three cases of $I_{\text {th }}$ is the same. We truncate the process into three groups: $\mathrm{A}, \mathrm{B}$, and C. In each group, we can see that 


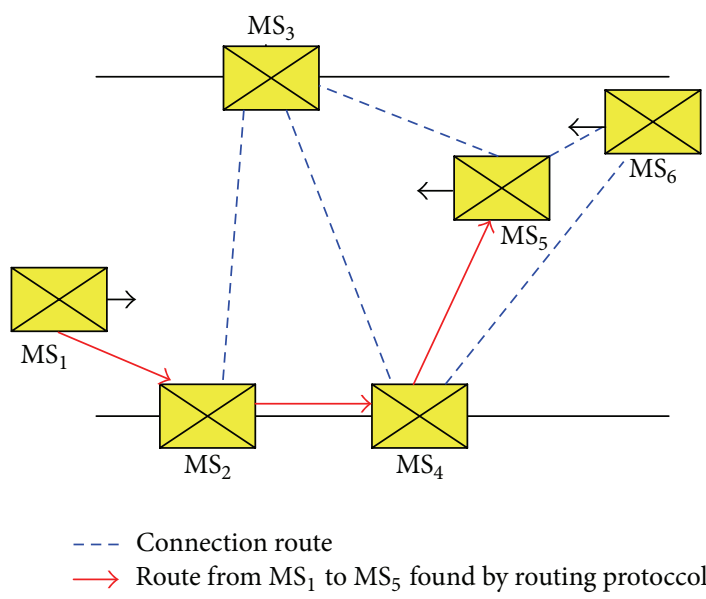

FIgURE 8: An example of the route from $\mathrm{MS}_{1}$ to $\mathrm{MS}_{5}$.

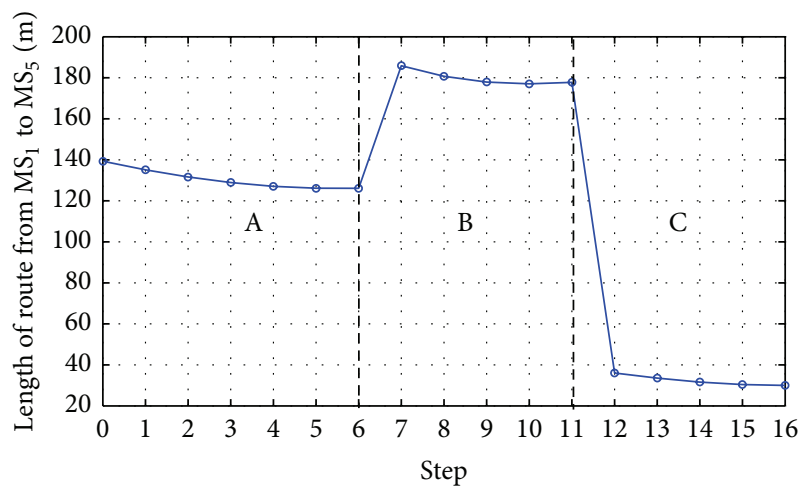

Figure 9: Length of route from $\mathrm{MS}_{1}$ to $\mathrm{MS}_{5}$.

lengths of routes seem to be the same. When the length of a route is high, it means that the number of intermediate nodes is also high. Therefore, the data is transmitted through many sections. In some sections, some sub-channels do not meet the QoS. Hence, the channel is not fullfilled, thus making the decrease of throughput. This conclusion is backedup from group: $\mathrm{A}$ and $\mathrm{B}$, where the lengths of routes are high and throughput is small in Figure 10. On the other hand, when the length of a route is small, the number of intermediate nodes is also small. Data can be directly transmitted from source to destination or through few intermediate nodes. In such case, the effect of interference, exposed node, and hidden node is reduced, and the channel is fullfilled. Consequently, the throughput of the network is significantly increased. It can be seen from group $C$ that the length of the route at each step is small and the throughputs would, therefore, be high. Throughput also depends on $I_{\text {th }}$ which decides a sub-channel be selected for transmission data or not. When $I_{\text {th }}$ is small, the probability that a sub-channel is selected is high. Hence, throughput is also high. The effect of $I_{\text {th }}$ is clear when the length of a route is high (in A and B groups) since it affects the selection of sub-channel in many intermediate sections. On the other hand, when mobile stations are close (in group C), the role of $I_{\text {th }}$ is not so important. Therefore, throughputs

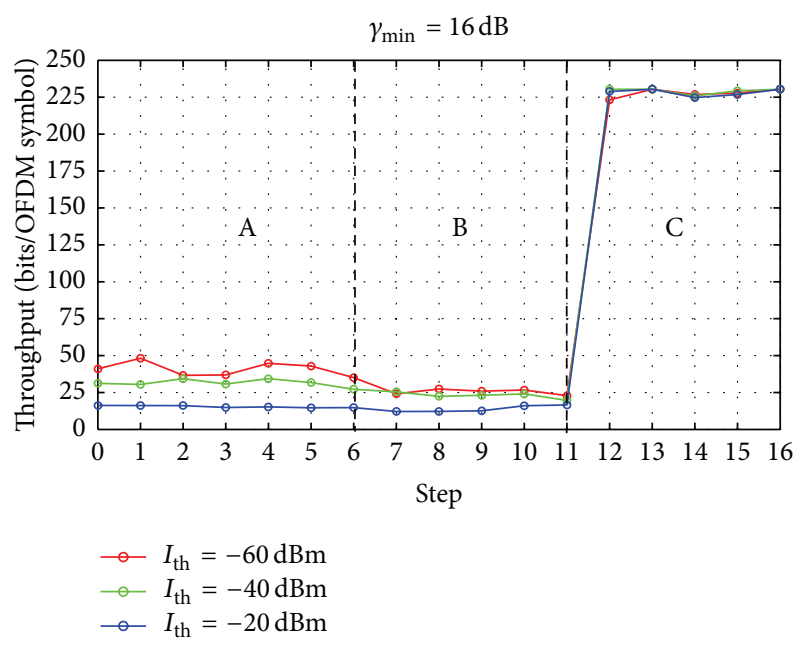

FIGURE 10: Throughput of the network when $\mathrm{MS}_{1}$ sends data to $\mathrm{MS}_{5}$.

seem to be similar for the three cases of $I_{\text {th }}$ of $-20,-40$, and $-60 \mathrm{dBm}$. It is clear that the throughput of the network depends on the distance between the source and destination nodes as well as $I_{\text {th }}$.

\subsection{Throughput of Multihop Ad Hoc Networks Based on the Proposed Routing Protocol}

5.3.1. Analysis Model. The performance of the proposed routing protocol is evaluated in this section. We implemented the routing protocol jointed with the MAC layer on the network as the one presented in Section 5.2. However, we consider the transmission data from $\mathrm{MS}_{1}$ to $\mathrm{MS}_{6}$. The channel allocation for the transmission data between nodes in the network is based on the proposed DSA algorithm. The threshold Channel_threshold is experimentally set to 42 . A mathematical method for deriving the optimal threshold will be proposed in our future work. $\gamma_{\min }$ and $I_{\text {th }}$ are set to $-80 \mathrm{dBW}$ and $16 \mathrm{~dB}$, respectively. An example of a route found by using the proposed routing protocol is drawn in Figure 11. In this state, the shortest route was found by the Dijkstra algorithm. The route, however, does not meet the requirement of the number of selected sub-channels. Hence, it was not selected for the transmitting data. On the other hand, our proposed protocol derived another route which has a longer length than the shortest route. Since the number of selected sub-channels of this route is larger than Channel_threshold, it was selected as the optimal route.

5.3.2. Numerical Results. The performance of the proposed routing protocol is depicted in Figures 12 and 13 where only conventional routing protocol is the principle routing based on the Dijkstra algorithm. It can be seen from these results that the throughput of the network depends on the distance between the source node and the destination node as in the conclusion in Section 5.2. In group A where the destination and the source node are far the number of intermediate nodes is high. Hence, the transmission significantly suffered from 


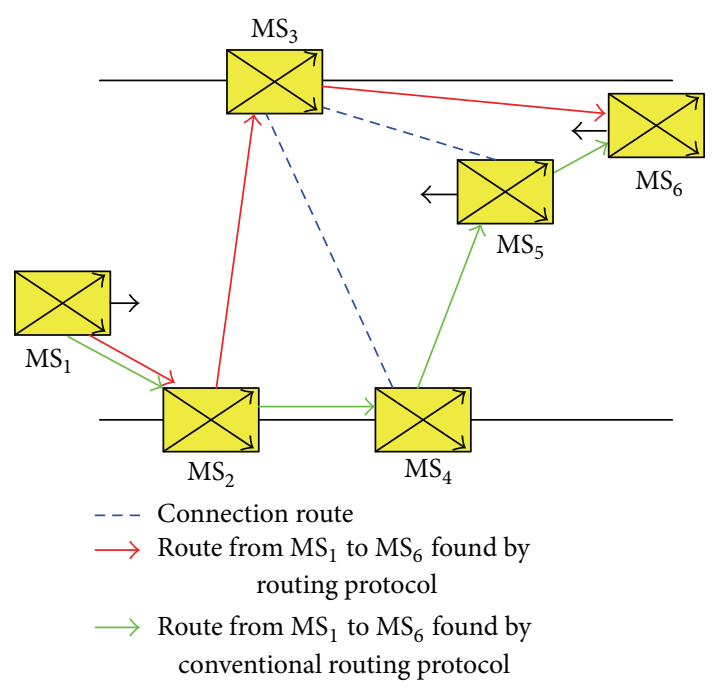

FIGURE 11: An example of a route from $\mathrm{MS}_{1}$ to $\mathrm{MS}_{6}$ found by using the proposed routing protocol.

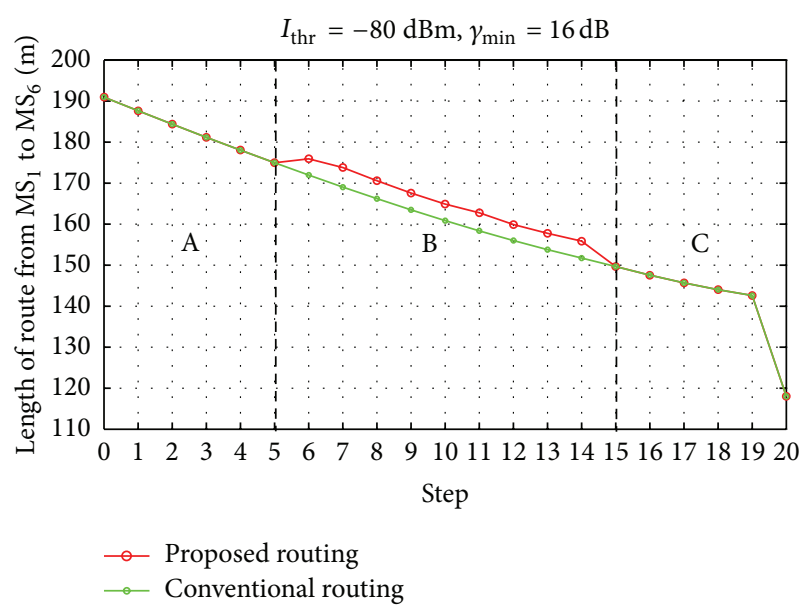

FIGURE 12: Length of the route from $\mathrm{MS}_{1}$ to $\mathrm{MS}_{6}$ using the proposed routing protocol and the conventional routing protocol.

the CCI as well as the hidden and exposed node problems. Consequently, the number of selected sub-channels of all available routes is smaller than Channel_threshold. Therefore, the proposed routing selected the shortest route as the optimal route to transmit the data and there is no difference of throughput the proposed routing protocol and the conventional one. On the other hand, in group $\mathrm{C}$ since the source and the destination are very close, they transmit its data directly or via a few of intermediate node. The effect of the CCI and the hidden and exposed node problems is considerably fell, thus making the increase of the number of selected subchannels. The number of selected sub-channels for all routes is larger than Channel_threshold. Therefore, as in group A, the proposed routing protocol selected the shortest route as the optimal route. In this group, the proposed routing and conventional routing protocols demonstrate the same performance. In group B, some routes have the number of selected sub-channel being larger than threshold, while one of the other routes is smaller than threshold. In some case,

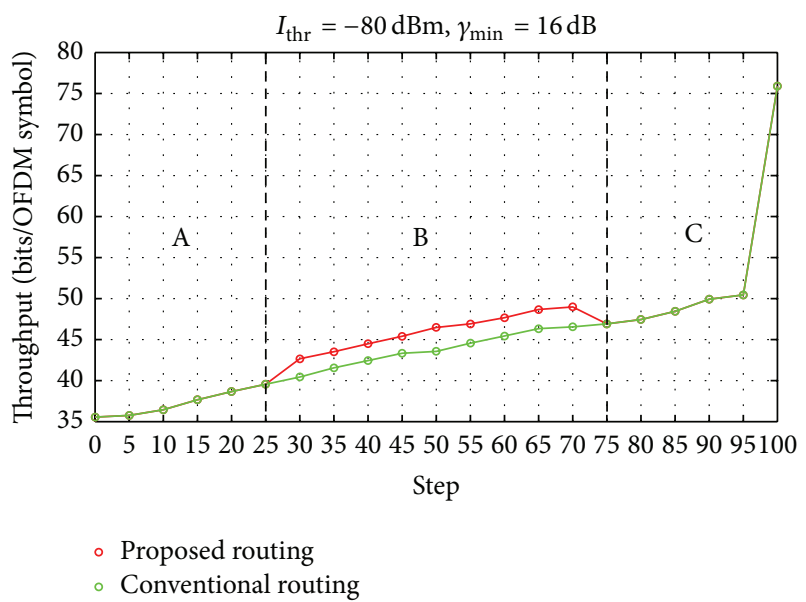

FIGURE 13: Throughput of the network using the proposed routing protocol and the conventional routing protocol.

the shortest route does not meet the requirement of the selected sub-channels; hence it is not selected as the optimal route. Our proposed find another route satisfy the requirement with longer length of route. Therefore, in this group, the proposed method outperforms the conventional routing protocol in terms of throughput of the network.

\section{Conclusions}

In this paper, we present a dynamic sub-channel assignment algorithm based on OFDM and a new routing protocol. A concept of multihop ad hoc networks based on the DSA algorithm is simulated with the proposed routing protocol. The proposed algorithm has an interference avoidance mechanism, so the throughput of the network can be maximized. The problems of cellular networks such as hidden node and exposed node are solved. A routing protocol which is jointed to the MAC protocol is proposed. The proposed routing protocol improves the throughput of multihop ad hoc networks as well as ensures the mobility, multihop of ad hoc and multihop networks. In the future, we will study the mathematical model for Channel_threshold parameter, network layer, and transport layer integration to improve and optimize QoS of multihop ad hoc networks.

\section{Acknowledgments}

This research is funded by the Vietnam National Foundation or Science and Technology Development (NAFOSTED) under the grant number 102.02-2011.15 and the Vietnam Ministry of Science and Technology (MOST) under the grant number KC01.05/11-15.

\section{References}

[1] G. Kulkarni and M. Srivastava, "Subcarrier and bit allocation strategies for OFDMA based wireless ad hoc networks," in Proceedings of the IEEE Global Telecommunications Conference (GLOBECOM '02), pp. 92-96, November 2002. 
[2] V. Venkataraman and J. J. Shynk, "Adaptive algorithms for OFDMA wireless ad hoc networks with multiple antennas," in Proceedings of the Conference Record of the 83th Asilomar Conference on Signals, Systems and Computers, pp. 110-114, November 2004.

[3] S. W. Kim and B.-S. Kim, "OFDMA-based reliable multicast MAC protocol for wireless ad-hoc networks," ETRI Journal, vol. 31, no. 1, pp. 83-85, 2009.

[4] V.-D. Nguyen, H. Haas, K. Kyamakya et al., "Decentralized dynamic sub-carrier assignment for OFDMA-based adhoc and cellular networks," IEICE Transactions on Communications, vol. E92-B, no. 12, pp. 3753-3764, 2009.

[5] J. M. Torrance and L. Hanzo, "Optimisation of switching levels for adaptive modulation in slow Rayleigh fading," Electronics Letters, vol. 32, no. 13, pp. 1167-1169, 1996.

[6] S. Skiena, Implementing Discrete Mathematics: Combinatorics and Graph Theory With Mathematica, Addison-Wesley, Reading, Mass, USA, 1990.

[7] V. D. Nguyen, P. E. Omiyi, and H. Haas, "Decentralised dynamic channel assignment for cellular OFDM/TDD networks," in Proceedings of the International OFDM Workshop (InOWo'05), vol. 3, pp. 255-2259, August 2005.

[8] S. W. Kim and B.-S. Kim, "OFDMA-based reliable multicast MAC protocol for wireless ad-hoc networks," ETRI Journal, vol. 31, no. 1, pp. 83-85, 2009.

[9] M. Stemick and H. Rohling, "OFDM-FDMA scheme for the uplink of a mobile communication system," Wireless Personal Communications, vol. 40, no. 2, pp. 157-170, 2007.

[10] C. Y. Wong, R. S. Cheng, K. B. Letaief, and R. D. Murch, "Multiuser OFDM with adaptive subcarrier, bit, and power allocation," IEEE Journal on Selected Areas in Communications, vol. 17, no. 10, pp. 1747-1758, 1999. 

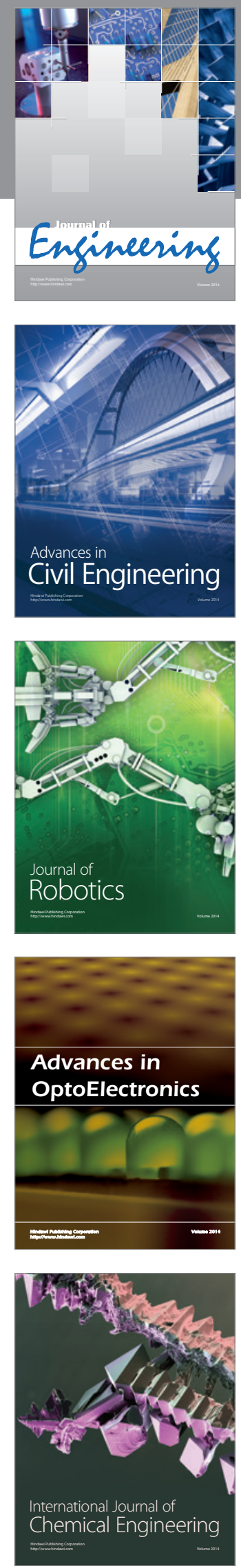

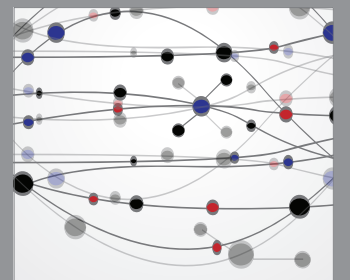

The Scientific World Journal
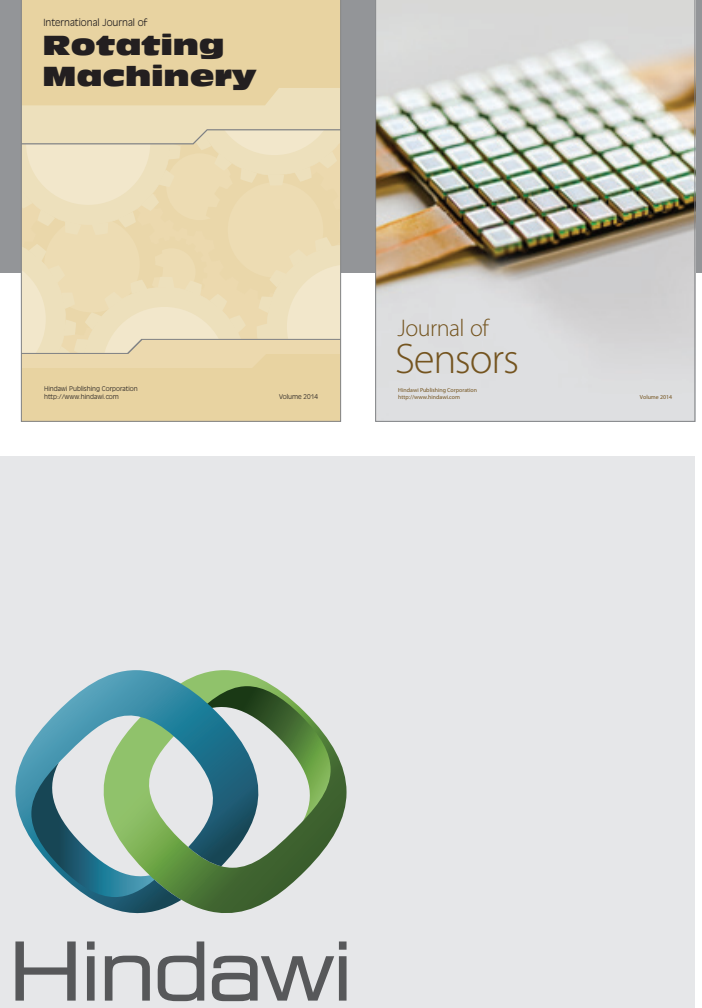

Submit your manuscripts at http://www.hindawi.com
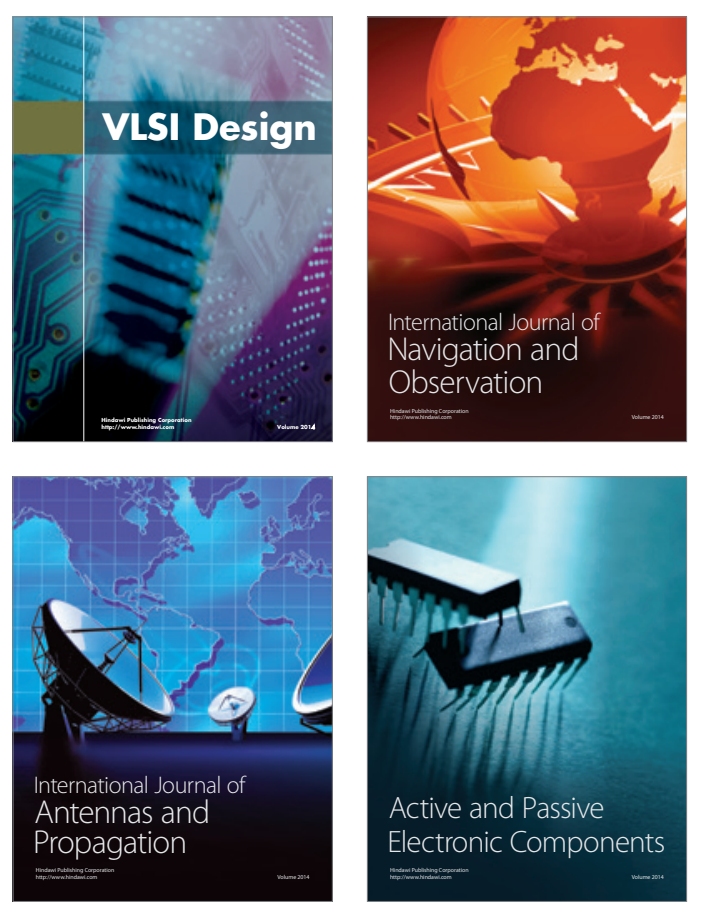
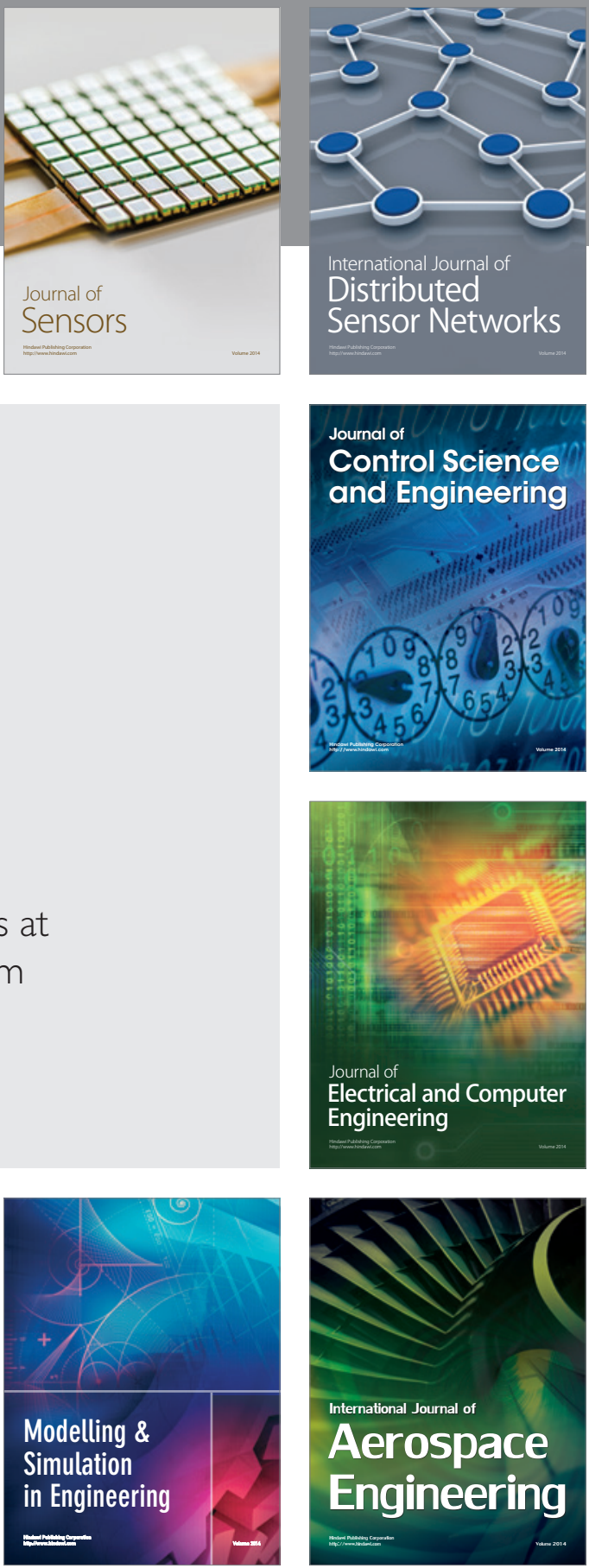

Journal of

Control Science

and Engineering
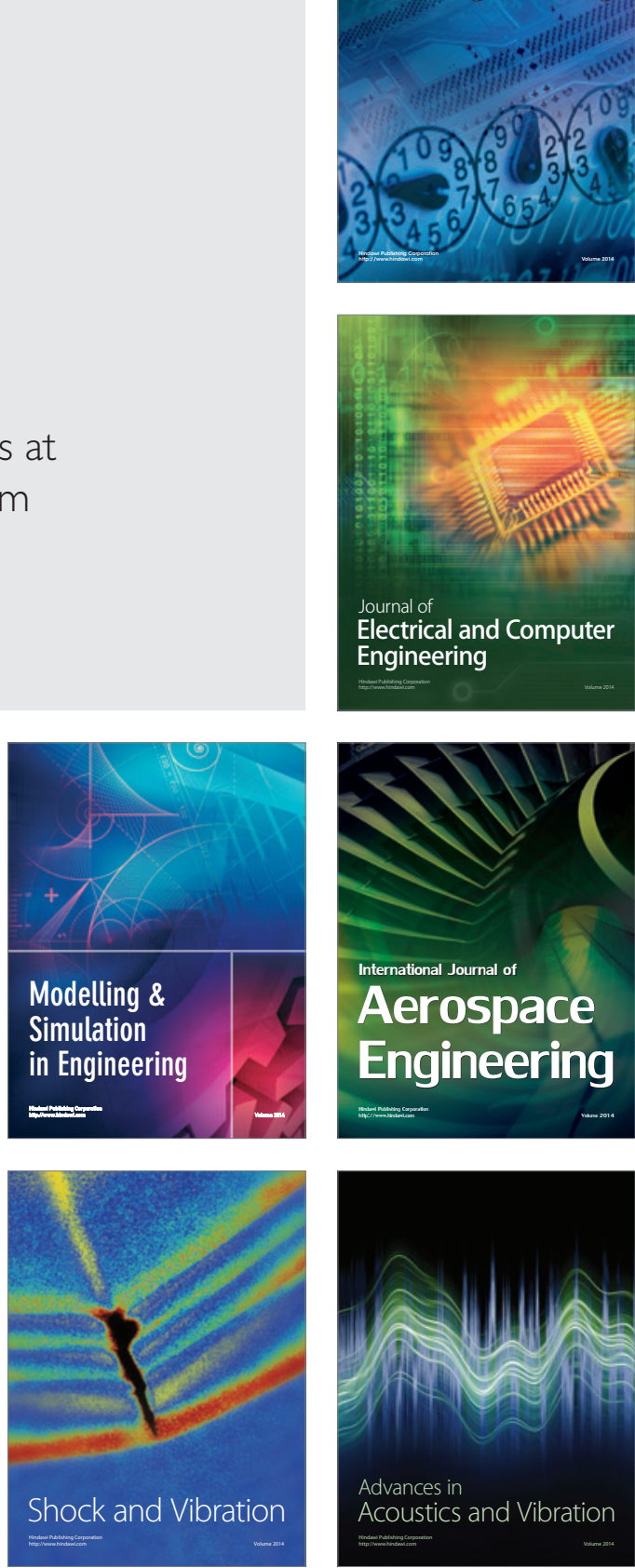